\title{
Hepatocyte growth factor inhibits hypoxia/reoxygenation-induced activation of xanthine oxidase in endothelial cells through the JAK2 signaling pathway
}

\author{
YING QIAN ZHANG $^{1 *}$, SHUN YING HU ${ }^{1 *}$, YUN DAI CHEN $^{1}$, MING ZHOU GUO $^{2}$ and SHAN WANG ${ }^{3}$ \\ Departments of ${ }^{1}$ Cardiology and ${ }^{2}$ Gastroenterology, Chinese PLA General Hospital, Beijing 100853; \\ ${ }^{3}$ Central Laboratory, Chinese PLA General Hospital, Beijing 100853, P.R. China
}

Received November 25, 2015; Accepted July 19, 2016

DOI: $10.3892 /$ ijmm.2016.2708

\begin{abstract}
Vascular endothelial cells (ECs) appear to be one of the primary targets of hypoxia/reoxygenation $(\mathrm{H} / \mathrm{R})$ injury. In our previous study, we demonstrated that hepatocyte growth factor (HGF) exhibited a protective effect in cardiac microvascular endothelial cells (CMECs) subjected to $\mathrm{H} / \mathrm{R}$ by inhibiting xanthine oxidase $(\mathrm{XO})$ by reducing the cytosolic $\mathrm{Ca}^{2+}$ concentration increased in response to $\mathrm{H} / \mathrm{R}$. The precise mechanisms through which HGF inhibits XO activation remain to be determined. In the present study, we examined the signaling pathway through which HGF regulates $\mathrm{Ca}^{2+}$ concentrations and the activation of $\mathrm{XO}$ during $\mathrm{H} / \mathrm{R}$ in primary cultured rat CMECs. CMECs were exposed to $4 \mathrm{~h}$ of hypoxia and $1 \mathrm{~h}$ of reoxygenation. The protein expression of $\mathrm{XO}$ and the activation of the phosphoinositide 3-kinase (PI3K), janus kinase 2 (JAK2) and p38 mitogen-activated protein kinase (p38 MAPK) signaling pathways were detected by western blot analysis. Cytosolic calcium $\left(\mathrm{Ca}^{2+}\right)$ concentrations and reactive oxygen species (ROS) levels were measured by flow cytometry. The small interfering RNA (siRNA)-mediated knockdown of XO inhibited the increase in ROS production induced by H/R. LY294002 and AG490 inhibited the $\mathrm{H} / \mathrm{R}$-induced increase in the production and activation of $\mathrm{XO}$. The PI3K and JAK2 signaling pathways were activated by $\mathrm{H} / \mathrm{R}$. The siRNA-mediated knockdown of PI3K and JAK2 also inhibited the increase in the production of $\mathrm{XO}$ protein.
\end{abstract}

Correspondence to: Dr Yun Dai Chen, Department of Cardiology, Chinese PLA General Hospital, 28 Fuxing Road, Beijing 100853, P.R. China

E-mail: dryundaic@163.com

\section{${ }^{*}$ Co-first authorship}

Abbreviation: ECs, endothelial cells; H/R, hypoxia/reoxygenation; $\mathrm{HGF}$, hepatocyte growth factor; $\mathrm{XO}$, xanthine oxidase; CMECs, cardiac microvascular endothelial cells; XDH, xanthine dehydrogenase; ROS, reactive oxygen species; siRNA, small interfering RNA

Key words: hepatocyte growth factor, hypoxia/reoxygenation, xanthine oxidase, endothelial cells, janus kinase 2
HGF inhibited JAK2 activation whereas it had no effect on PI3K activation. The siRNA-mediated knockdown of JAK2 prevented the increase in cytosolic $\mathrm{Ca}^{2+}$ induced by H/R . Taken together, these findings suggest that $\mathrm{H} / \mathrm{R}$ induces the production and activation of $\mathrm{XO}$ through the JAK2 and PI3K signaling pathways. Furthermore, HGF prevents $\mathrm{XO}$ activation following $\mathrm{H} / \mathrm{R}$ primarily by inhibiting the JAK2 signaling pathway and in turn, inhibiting the increase in cytosolic $\mathrm{Ca}^{2+}$.

\section{Introduction}

Given their unique locations, vascular endothelial cells (ECs) appear to be one of the primary targets of hypoxia/reoxygenation $(\mathrm{H} / \mathrm{R})$ injury $(1,2)$. The generation of and substantially increased levels of reactive oxygen species (ROS) (3), have been widely implicated in EC injury $(4,5)$. Xanthine oxidoreductase (XOR) exists as two distinct enzyme forms: xanthine dehydrogenase (XDH) and xanthine oxidase (XO). $\mathrm{XDH}$ requires $\mathrm{NAD}^{+}$to reduce hypoxanthine to xanthine. $\mathrm{XO}$ requires $\mathrm{O}_{2}$ for purine oxidation, thereby generating ROS (6). $\mathrm{XO}$ has been identified as a source of ROS in atherosclerosis (7), coronary artery disease (8) and heart failure (9). An in vivo study showed that $\mathrm{XDH}$ expression is increased during $\mathrm{H} / \mathrm{R}$ in rat kidneys (10). Another in vitro study demonstrated that the XDH-to-XO conversion is stimulated by hydrogen peroxide and calcium $\left(\mathrm{Ca}^{2+}\right)$ in bovine aortic endothelial cells (11). Furthermore, the p38 mitogen-activated protein kinase (p38 MAPK), janus kinase 2 (JAK2) and signal transducers and activators of transcription (STAT) signaling pathways are reportedly involved in the process of $\mathrm{XO}$ activation and $\mathrm{XDH}$-to-XO conversion during hypoxia in pulmonary microvascular endothelial cells $(12,13)$.

Hepatocyte growth factor (HGF) has been found to promote survival, proliferation and morphogenesis by activating its receptor cMet. HGF enhances the migration of epithelial cells following acute kidney injury (14). HGF has also been found to regulate neovascularization in developing fat pads (15). In our previous study, we demonstrated that HGF may inhibit XO production and activation by reducing the cytosolic $\mathrm{Ca}^{2+}$ concentration increased in response to $\mathrm{H} / \mathrm{R}$; thus, HGF protects cardiac microvascular endothelial cells (CMECs) from H/R-induced ROS production and H/R-induced cell apoptosis (16). However, 
the signaling mechanisms through which HGF regulates cytosolic $\mathrm{Ca}^{2+}$ concentrations and $\mathrm{XO}$ activation in CMECs under conditions of $\mathrm{H} / \mathrm{R}$ remain to be elucidated. In the present study, we examined the signaling pathway through which HGF regulates $\mathrm{Ca}^{2+}$ concentrations and the activation of $\mathrm{XO}$ during $\mathrm{H} / \mathrm{R}$ in primary cultured rat CMECs.

\section{Materials and methods}

Isolation and culture of CMECs. A total of 80 five-to-seven day old Sprague-Dawley (SD) rats weighing 12-16 g, were purchased from the Experimental Animal Center of the Chinese PLA General Hospital (Beijing, China). Ethics approval was obtained from the Ethics Committee of the Experimental Animal Center of the Chinese PLA General Hospital (approval no. SCXK20120001). All procedures were performed according to the National Institutes of Health Guideline for the Care and Use of Laboratory Animals. The rats were sacrificed with an overdose of isoflurane and the hearts were removed. The enzyme dissociation method based on the one described by Nishida et al was used (17). The cells were collected and resuspended in Dulbecco's modified Eagle's medium (DMEM) (\#12100046; Gibco, Grand Island, NY, USA) supplemented with 20\% fetal bovine serum (FBS) (\#YS-OS-001091; HyClone, Logan, UT, USA) and then seeded in $25 \mathrm{~cm}^{2}$ polystyrene flasks. Cell purity was identified by morphological (18) and immunohistochemical characteristics (4): the CMEC monolayer displayed a uniform 'cobblestone' morphology and positive immunohistochemical assays of factor VIII (\#ab61910; Abcam, Cambridge, MA, USA) and CD31 (\#sc71873; Santa Cruz Biotechnology, Inc., Santa Cruz, CA, USA) (>95\%).

$H / R$ procedure and drug treatment. The $\mathrm{H} / \mathrm{R}$ procedure was achieved by subjecting the cells to $4 \mathrm{~h}$ of hypoxia and $1 \mathrm{~h}$ of reoxygenation. For hypoxic exposure, the cells were incubated in D-Hank's solution (in mM: $136.89 \mathrm{NaCl}, 5.37 \mathrm{KCl}, 4.166$ $\mathrm{NaHCO}_{3}, 0.44 \mathrm{KH}_{2} \mathrm{PO}_{4}, 0.338 \mathrm{Na}_{2} \mathrm{HPO}_{4}, \mathrm{pH} 7.3-7.4$ at $37^{\circ} \mathrm{C}$ ) saturated with $95 \% \mathrm{~N}_{2}$ and $5 \% \mathrm{CO}_{2}$. The $\mathrm{pH}$ was adjusted to 6.8 to mimic ischemic conditions. The cells were placed in a hypoxic incubator (Invivo2; Ruskinn Technology Ltd., Pencoed, UK) that was equilibrated with $95 \% \mathrm{~N}_{2}$ and 5\% $\mathrm{CO}_{2}$. Ambient $\mathrm{O}_{2}$ levels in the incubator were monitored by an $\mathrm{O}_{2}$ analyzer (series-2000; Alpha Omega Instruments, Cumberland, RI, USA). Following hypoxic exposure, the culture medium was rapidly replaced with DMEM together with $1 \%$ FBS to initiate the reoxygenation procedure (19-21).

For drug treatment, the CMECs were pre-incubated with $30 \mu \mathrm{M}$ AG490 (\#S1509), $30 \mu \mathrm{M}$ SB203580 (\#S1863), $1 \mu \mathrm{M}$ LY294002 (\#S1737) (all from Beyotime Biotech, Jiangsu, China) or 10 or 20 ng/ml HGF (\#294-HG-005; R\&D Systems, Inc., Minneapolis, MN, USA) for $30 \mathrm{~min}$ prior to exposure to hypoxic conditions.

Measurement of ROS generation. Following the different treatments, the CMECs were incubated with $2 \mathrm{mM}$ DCFH-DA (\#287810; Sigma-Aldrich, St. Louis, MO, USA) for $20 \mathrm{~min}$ at $37^{\circ} \mathrm{C}$ in a $5 \% \mathrm{CO}_{2}$ incubator. The cells were washed and resuspended in phosphate-buffered saline (PBS) at a concentration of $1 \times 10^{6}$ cells $/ \mathrm{ml}$. DCF fluorescence was analyzed using a flow cytometer (Becton-Dickinson, Mountainview, CA, USA) at the excitation and emission wavelengths of 514 and $525 \mathrm{~nm}$, respectively. Untreated cells served as controls. The amount of ROS was calculated as the fold-increase in DCF fluorescence compared with the controls (22).

Small interfering RNA (siRNA) transfection experiments. The transient transfection of CMECs with $50 \mathrm{nM}$ siRNA oligonucleotide was performed using Lipofectamine RNAiMAX reagent (\#13778030; Invitrogen, Dublin, Ireland). The cells were seeded in 6 -well plates $\left(2 \times 10^{5}\right.$ cells/well) for these studies. The following siRNA sequences were used: rat XDH siRNA, 5'-CCACCUCCAAGAUUCAUAUTT-3'; rat phosphoinositide 3-kinase (PI3K) siRNA, 5'-GCAGCCAGCU CUGAUAAUATT-3'; rat JAK2 siRNA, 5'-GCCCUAAGGAC UUCAACAATT-3' and rat p38 MAPK siRNA, 5'-GGACCUC CUUAUAGACGAATT-3'. These siRNAs and their non-targeting sequences (negative controls) were synthesized by GenePharma Co., Ltd. (Shanghai, China). After $48 \mathrm{~h}$ of transfection, the CMECs were subjected to the H/R procedure. Finally, the cells were harvested for other experiments.

Measurement of cytosolic $\mathrm{Ca}^{2+}$. The CMECs were loaded with fluo-3 (\#F23915; Invitrogen, Carlsbad, CA, USA) in $1 \%$ working solution at $37^{\circ} \mathrm{C}$ for $30 \mathrm{~min}$. The cells were washed three times with $\mathrm{Ca}^{2+}$-free PBS to remove extracellular fluo-3 AM, and then resuspended in PBS at a concentration of $1 \times 10^{6}$ cells $/ \mathrm{ml}$. The cells were analyzed by flow cytometry (Becton-Dickinson) at an excitation wavelength of $488 \mathrm{~nm}$, and an emission wavelength of $530 \mathrm{~nm}$ (23). The untreated cells served as controls.

Western blot analysis. The CMECs were homogenized in RIPA lysis buffer (\#P0013C; Beyotime) containing 1X Phosphatase Inhibitor Cocktail (\#5870S; Cell Signaling Technology, Beverly, MA, USA) and $1 \mu \mathrm{g} / \mathrm{ml}$ each of aprotinin (A1153; Sigma-Aldrich) and leupeptin (\#L2884; Sigma-Aldrich). Forty micrograms of protein was separated by sodium dodecyl sulfate-polyacrylamide gel electrophoresis (SDS-PAGE), transferred to polyvinylidene difluoride (PVDF) membranes, and then probed with antibodies for XO (\#ab109235; Abcam), phospho-PI3 kinase p85 (Tyr458)/p55 (Tyr199) (\#4228), PI3 kinase p85 (19H8) (\#4257), phospho-p38 MAP kinase (Thr180/Thr182) (\#4631), p38 MAPK (D13E1) (\#8690), JAK2 (D2E12) (\#3230) and phospho-JAK2 (Tyr1007/1008) (C80C3) (\#3776) (all from Cell Signaling Technology, Beverly, MA, USA). The same membranes were reprobed with an antibody for tubulin (\#AT819; Beyotime). The blotting film was quantified using a scanner and a densitometry program (ImageJ; https://imagej.nih.gov/ij/index.html) (24). To quantify the phospho-specific signal in the activated samples, the background was subtracted and the band was normalized to the amount of tubulin or total target protein in the lysate.

Statistical analysis. Statistical comparisons were performed using the paired, two-tailed Student's t-test for experiments consisting of two groups only, with one-way ANOVA and a multiple comparison method for experiments consisting of more than two groups. A p $<0.05$ was considered to indicate a statistically significant difference. Data are presented as the means $\pm \mathrm{SE}$. 

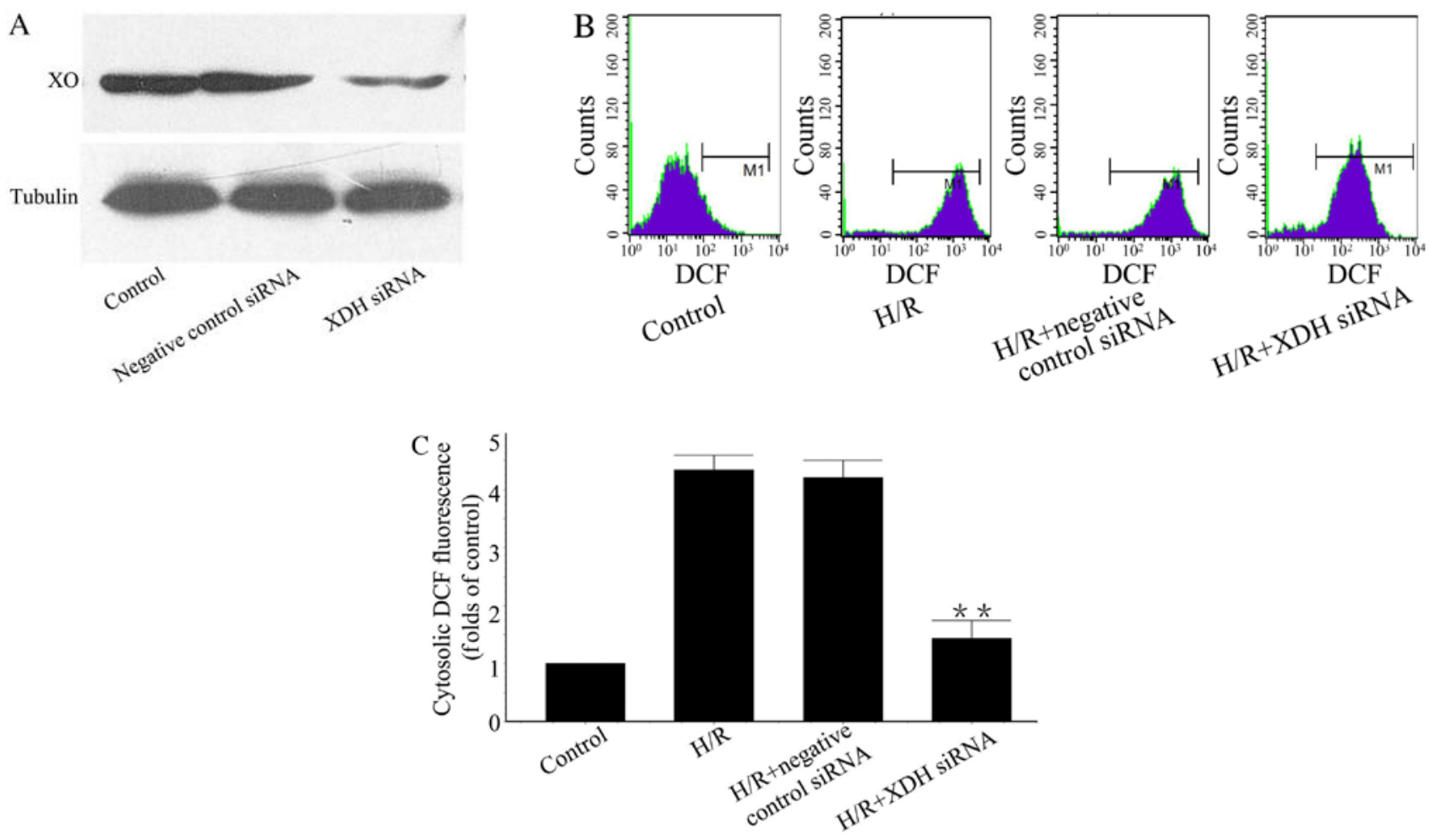

Figure 1. Knockdown of xanthine oxidase (XO) attenuates the hypoxia/reoxygenation (H/R)-induced production of ROS. (A) Representative western blot of XO and tubulin expression in cardiac microvascular endothelial cells (CMECs) transfected with either no siRNA, negative control siRNA or xanthine dehydrogenase (XDH) siRNA. (B) Representative data for flow cytometric analysis of DCFH-DA-stained CMECs transfected with negative control siRNA or XDH siRNA, and compared with the H/R group. (C) Summary data ( $n=3$ biological replicates) of flow cytometric analysis of DCFH-DA-stained CMECs under conditions of $\mathrm{H} / \mathrm{R}$ transfected with negative control siRNA or XDH siRNA, and compared with the H/R group. ** p $<0.01$ vs. H/R group.

A

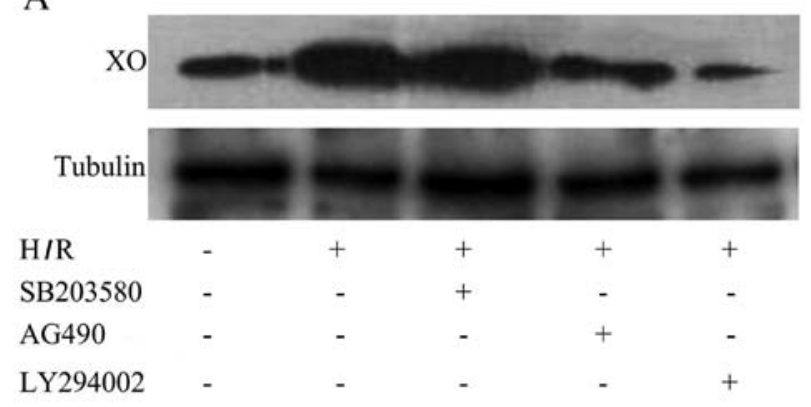

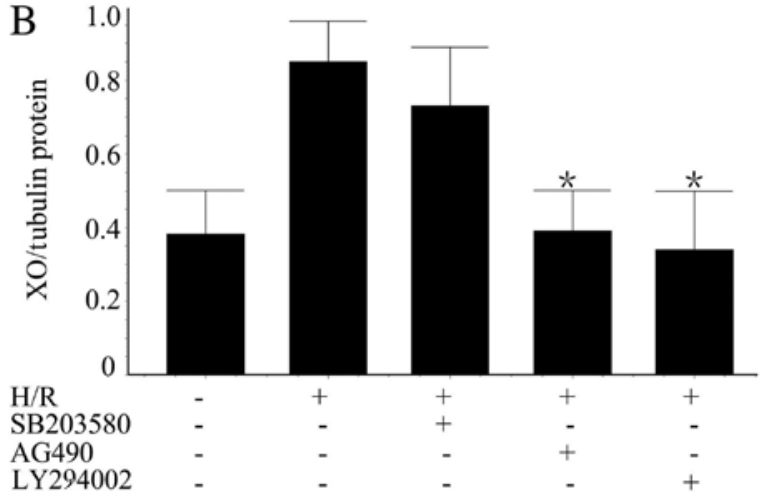

Figure 2. PI3K and JAK2 signaling pathways are involved in the production and activation of xanthine oxidase (XO). (A) Representative western blot of XO and tubulin expression in cardiac microvascular endothelial cells (CMECs) following hypoxia/reoxygenation (H/R). LY294002 and AG490 significantly decreased the increase in the XO protein level induced by H/R. (B) Summary data ( $n=3$ biological replicates) of western blot analysis of XO and tubulin expression in CMECs following H/R. LY294002, SB203580 and AG490 treatment group compared with H/R group. *p<0.05 vs. H/R group.

\section{Results}

XO plays a key role in the H/R-induced production of ROS. In our previous study, the production of ROS following H/R was significantly attenuated by allopurinol (20 and $40 \mu \mathrm{mol} / \mathrm{l})$, an inhibitor of XO (16). In the present study, the expression of XO was knocked down by XDH siRNA (Fig. 1A). Four hours of hypoxia increased intracellular DCF fluorescence compared with normoxia (control group). The transfection of $\mathrm{XDH}$ siRNA attenuated the increased production of ROS following H/R (Fig. 1B and C).
PI3K and JAK2 pathways are involved in the production and activation of XO. To determine whether PI3K, p38 MAPK or JAK2 signaling pathways are involved in the production and activation of $\mathrm{XO}$, we examined the effect of PI3K inhibitor LY294002, p38 MAPK inhibitor SB203580 and JAK2 inhibitor AG490 on the production and activation of XO. Pre-treatment with LY294002 and AG490 inhibited H/R-mediated XO production (Fig. 2A and B). The phosphorylation of PI3K and JAK2 was significantly increased following H/R as compared with the normoxia controls (Fig. 2C and D). However, the phosphorylation of p38 MAPK was not found to be increased 

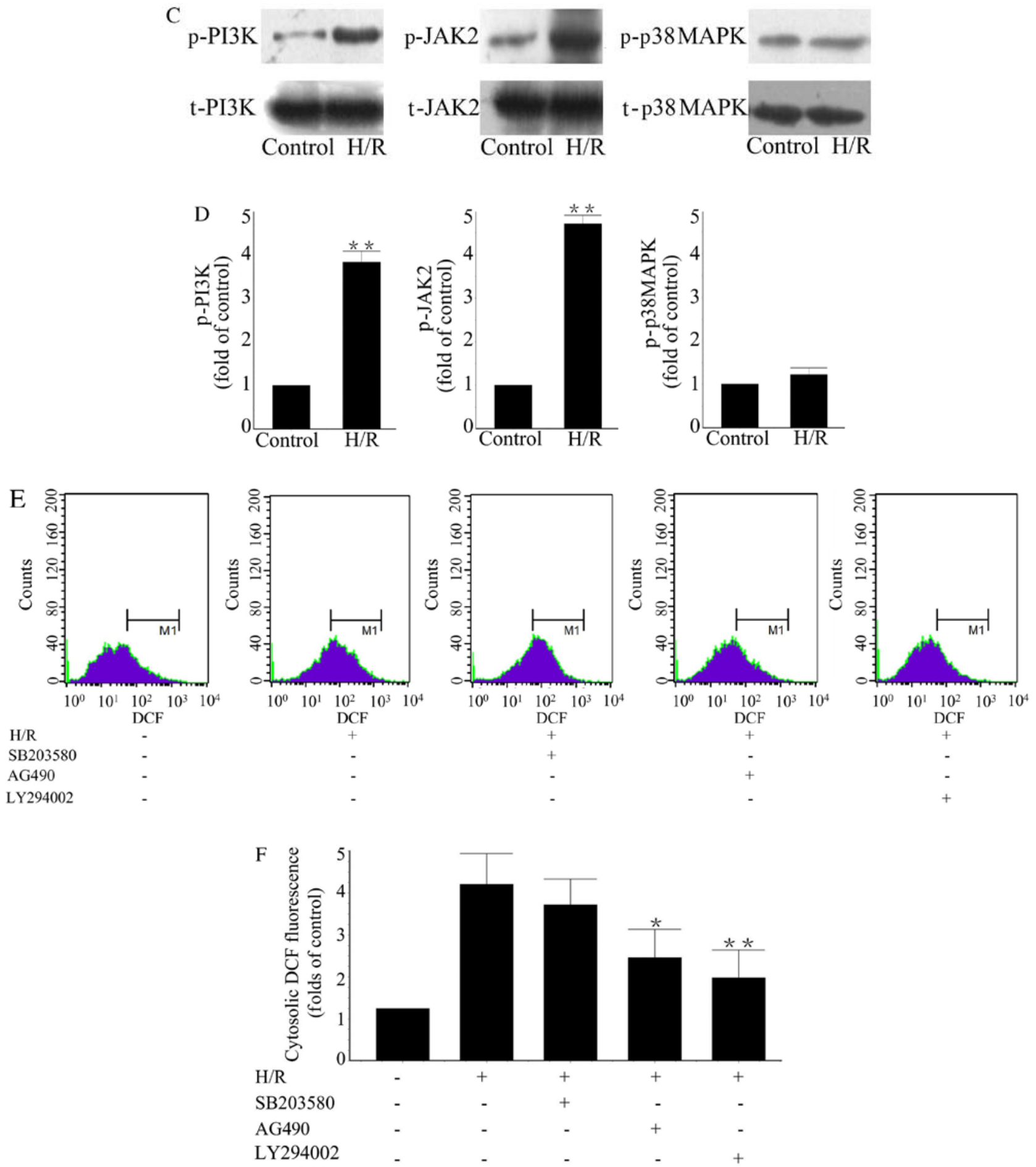

Figure 2. Continued. PI3K and JAK2 signaling pathways are involved in the production and activation of xanthine oxidase (XO). (C) Representative western blots of phosphorylated (p-)PI3K, total (t-)PI3K, p-JAK2, t-JAK2, p-p38 MAPK and t-p38 MAPK expression in cardiac microvascular endothelial cells (CMECs) following hypoxia/reoxygenation $(\mathrm{H} / \mathrm{R})$. H/R activates the PI3K and JAK2 signaling pathways but not the p38 MAPK signaling pathway. (D) Summary data ( $\mathrm{n}=3$ biological replicates) of western blot analysis of p-PI3K, t-PI3K, p-JAK2, t-JAK2, p-p38 MAPK and t-p38 MAPK expression in CMECs. *" $<<0.01$ vs. control group. (E) Representative data for flow cytometric analysis of DCFH-DA-stained CMECs following H/R. LY294002, SB203580 and AG490 treatment groups compared with H/R group. (F) Summary data ( $n=3$ biological replicates) of flow cytometric analysis of DCFH-DA-stained CMECs following H/R. LY294002 $(1 \mu \mathrm{M})$, SB203580 $(30 \mu \mathrm{M})$ and AG490 $(30 \mu \mathrm{M})$ treatment groups compared with H/R group. "p $<0.05 \mathrm{vs.} \mathrm{H/R}$ group; ${ }^{* *} \mathrm{p}<0.01$ vs. H/R group.

after H/R (Fig. 2C and D). ROS production following H/R was also partly blocked by LY294002 and AG490, respectively (Fig. 2E and F). These data indicated that $\mathrm{XO}$ activation induced by $\mathrm{H} / \mathrm{R}$ in CMECs is mediated through the PI3K and JAK2 signaling pathways rather than the p38 MAPK signaling pathway.
PI3K siRNA and JAK2 siRNA downregulate the production and activation of $X O$. To further confirm the involvement of $\mathrm{PI} 3 \mathrm{~K}$ and JAK2 signaling pathways in the production and activation of XO following H/R, CMECs were transfected with either PI3K siRNA, JAK2 siRNA or p38 MAPK siRNA to introduce knockdown (Fig. 3A). When the expression of 
A p38MAPK

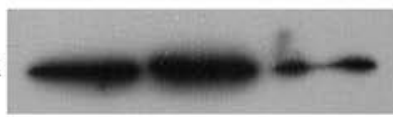

Tubulin

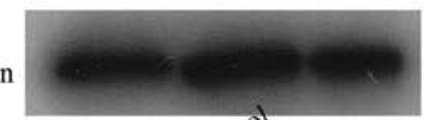

PI3K

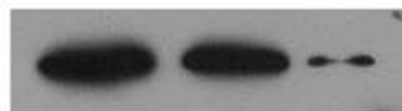

Tubulin

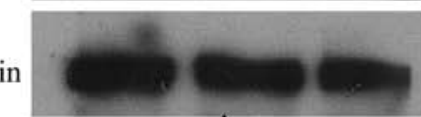

JAK2

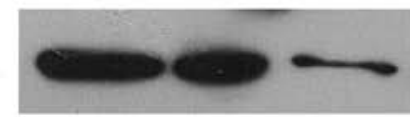

Tubulin

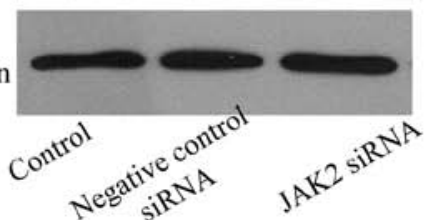

B

$\mathrm{H} / \mathrm{R}$

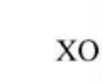

Tubulin
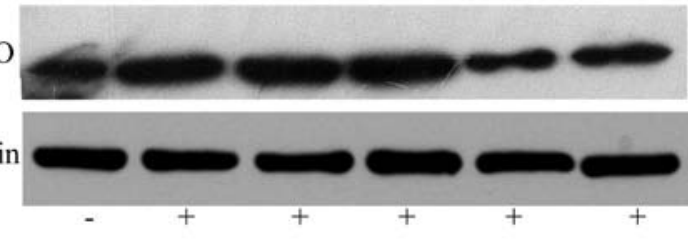

Negative control siRNA p38MAPK siRNA PI3K siRNA

JAK2 siRNA
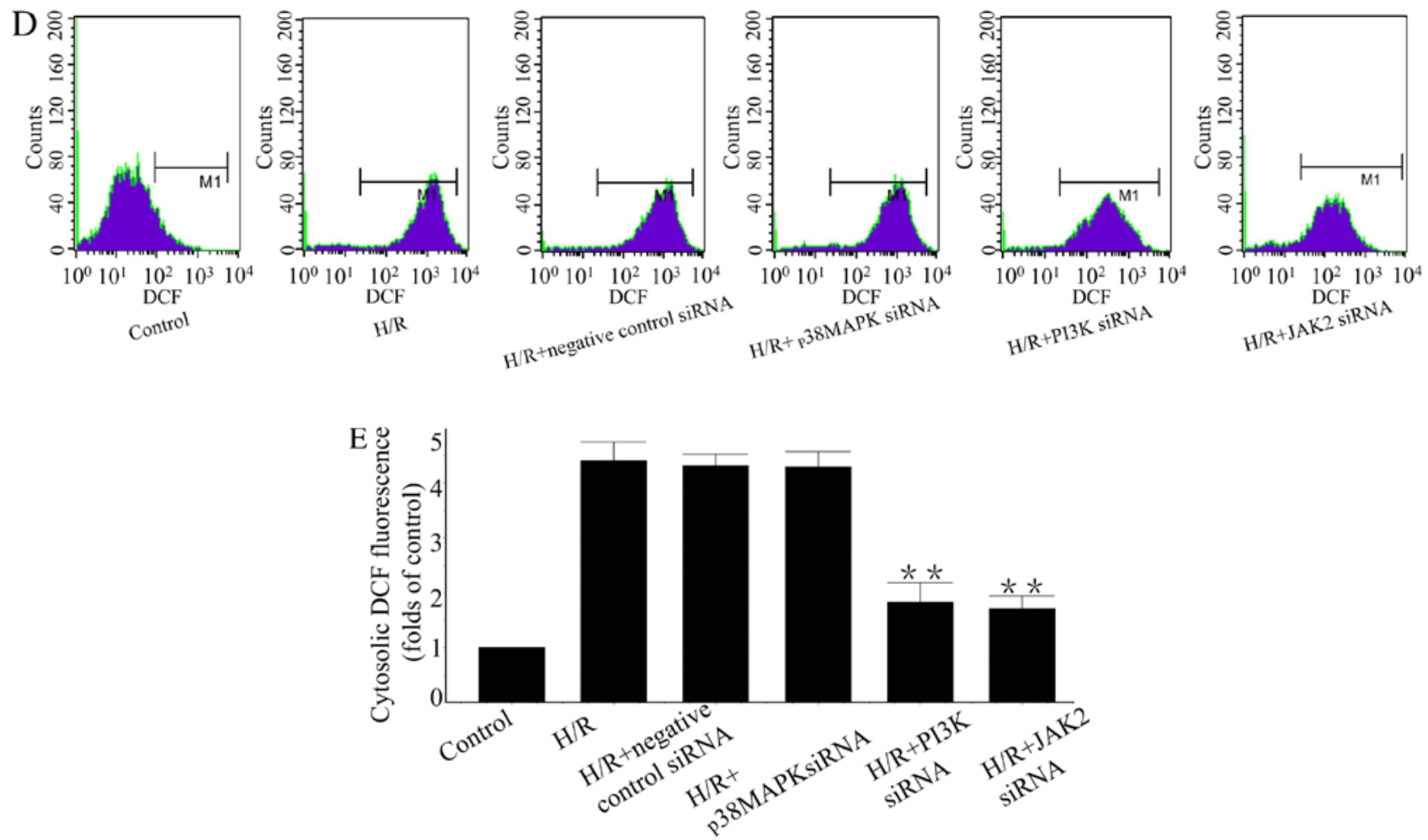

Figure 3. PI3K siRNA and JAK2 siRNA downregulate the production and activation of xanthine oxidase (XO). (A) Representative western blots of p38 MAPK, PI3K, JAK2 and tubulin expression in cardiac microvascular endothelial cells (CMECs). (B) Representative western blot of XO and tubulin expression in CMECs following hypoxia/reoxygenation (H/R). PI3K siRNA-, p38 MAPK siRNA-, JAK2 siRNA- and negative control siRNA-transfected groups compared with H/R group. (C) Summary data ( $\mathrm{n}=3$ biological replicates) for western blot analysis of $\mathrm{XO}$ and tubulin expression in CMECs following H/R. PI3K siRNA-, p38 MAPK siRNA-, JAK2 siRNA- or negative control siRNA-transfected groups compared with H/R group. ${ }^{* *}$ p $<0.01$ vs. H/R group. (D) Representative flow cytometric analysis of DCFH-DA-stained CMECs after H/R. PI3K siRNA-, p38 MAPK siRNA-, JAK2 siRNA- or negative control siRNA-transfected groups compared with H/R group. (E) Summary data ( $\mathrm{n}=3$ biological replicates) for flow cytometric analysis of DCFH-DA-stained CMECs after H/R. PI3K siRNA-, p38 MAPK siRNA-, JAK2 siRNA- or negative control siRNA-transfected groups compared with H/R group. ** $<<0.01$ vs. H/R group.

PI3K and JAK2 was inhibited by their respective siRNAs, the $\mathrm{H} / \mathrm{R}$-induced increase in $\mathrm{XO}$ production was downregulated (Fig. 3B and C). ROS production following H/R was also partly blocked by PI3K siRNA and JAK2 siRNA, respectively (Fig. 3D and E) However, p38 MAPK siRNA did not exert similar effects. These data further confirmed that the 

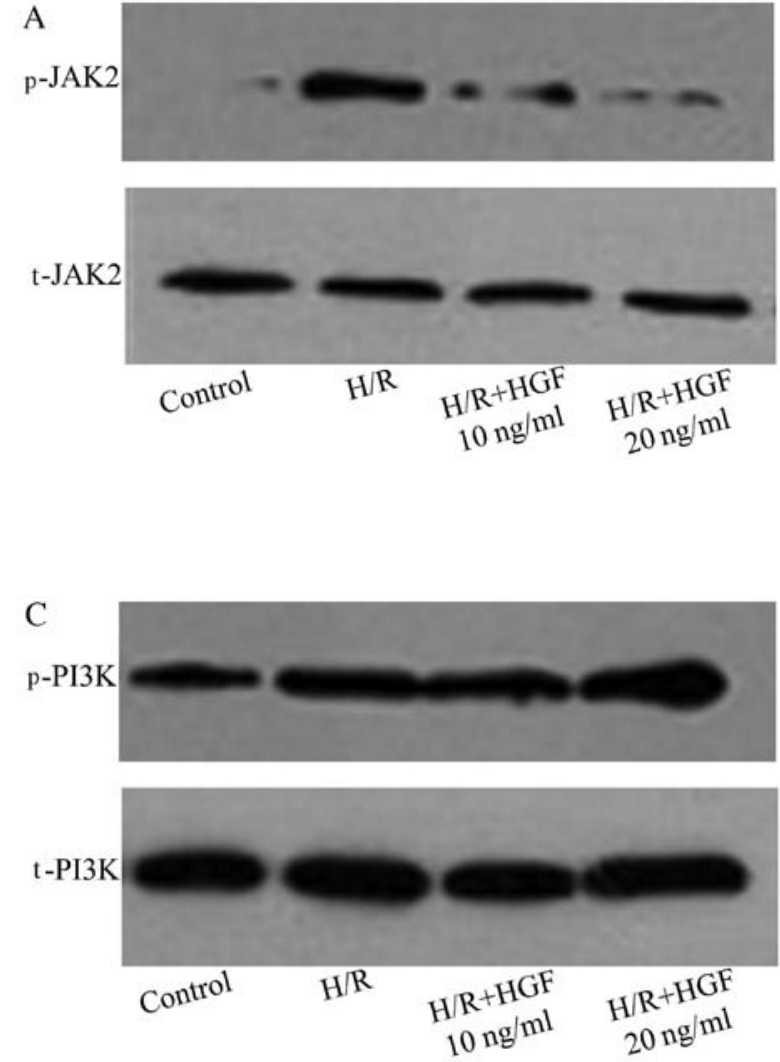
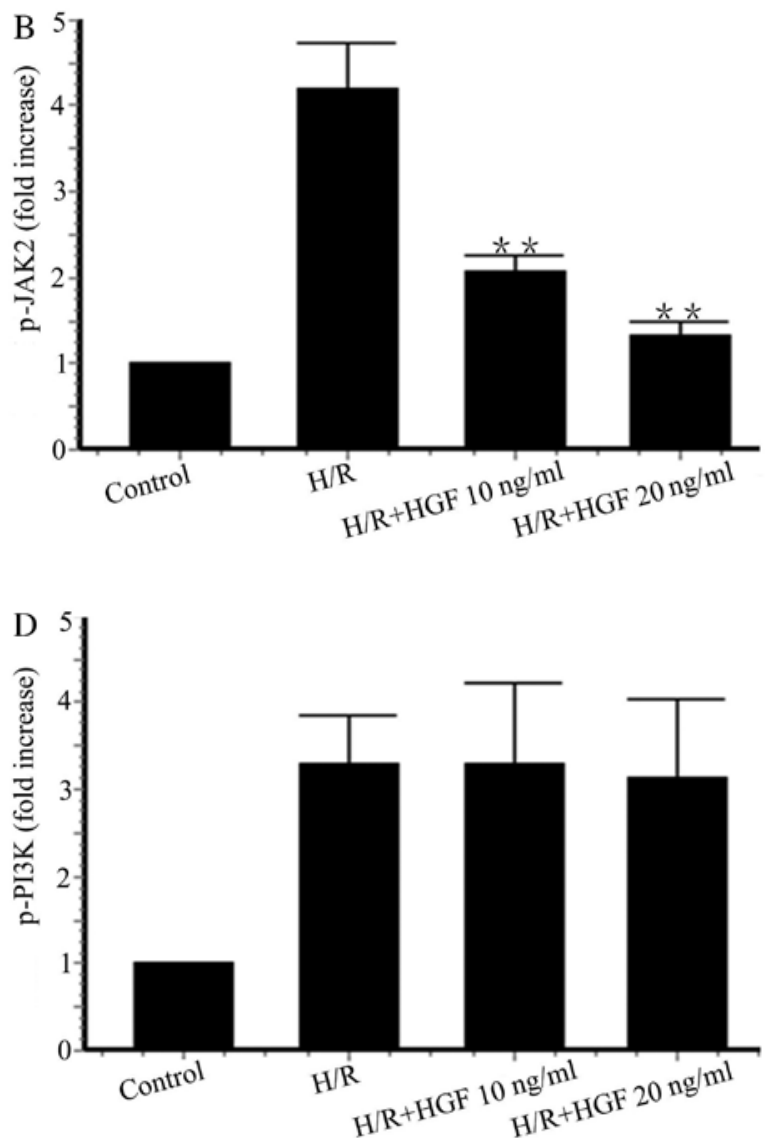

Figure 4. Hepatocyte growth factor (HGF) inhibits activation of the JAK2 signaling pathway but has no effect on the PI3K signaling pathway. (A) Representative western blot of phosphorylated (p-)JAK2 and total (t-)JAK2 expression in cardiac microvascular endothelial cells (CMECs) following hypoxia/reoxygenation (H/R). HGF significantly decreased the H/R-induced activation of the JAK2 pathway. (B) Summary data (n=3 biological replicates) for western blot analysis of p-JAK2 and t-JAK2 in CMECs after H/R. HGF (10 and $20 \mathrm{ng} / \mathrm{ml})$ treatment groups compared with H/R group. ${ }^{* *}$ p<0.01 vs. H/R group. (C) Representative western blot of p-PI3K and t-PI3K expression in CMECs after H/R. HGF had no effect on the PI3K signaling pathway. (D) Summary data ( $n=3$ biological replicates) for western blot analysis of $\mathrm{p}-\mathrm{PI} 3 \mathrm{~K}$ and $\mathrm{t}-\mathrm{PI} 3 \mathrm{~K}$ expression in CMECs following H/R.

production and activation of $\mathrm{XO}$ induced by $\mathrm{H} / \mathrm{R}$ in CMECs is mediated through the PI3K and JAK2 signaling pathways rather than the 38 MAPK signaling pathway.

HGF inhibits JAK2 activation but not PI3K activation. The phosphorylation of JAK 2 and PI3K was evaluated by western blot analysis in CMECs pre-treated with HGF (10 and $20 \mathrm{ng} / \mathrm{ml}$ ). The phosphorylation of JAK2 induced by H/R was inhibited by HGF (Fig. 4A and B). However, the phosphorylation of $\mathrm{PI} 3 \mathrm{~K}$ induced by $\mathrm{H} / \mathrm{R}$ was unaffected by HGF (Fig. 4C and D). These findings suggest that HGF inhibited the activation and production of $\mathrm{XO}$ through the JAK2 signaling pathway.

JAK2 siRNA downregulates the concentration of cytosolic calcium. In our previous study, we found that HGF inhibits $\mathrm{XO}$ activation by reducing cytosolic $\mathrm{Ca}^{2+}$ concentrations induced by H/R (16). We further studied whether the JAK2 signaling pathway regulates cytosolic $\mathrm{Ca}^{2+}$ concentrations. JAK 2 knockdown was achieved by JAK 2 siRNA, and resulted in a reduction in the cytosolic $\mathrm{Ca}^{2+}$ concentration induced by H/R (Fig. 5). Taken together, these findings suggest that HGF reduced cytosolic $\mathrm{Ca}^{2+}$ concentrations by inhibiting JAK 2 phosphorylation.

\section{Discussion}

Excessive oxidative stress is believed to be an important contributor to H/R injury. In our previous study, we revealed that HGF protects CMECs from H/R-induced apoptosis by reducing ROS production (16). The findings of the present study indicate that $\mathrm{H} / \mathrm{R}$ increased DCF oxidation. DCF detects $\mathrm{H}_{2} \mathrm{O}_{2}$ but does not detect superoxide (25). However, $\mathrm{H}_{2} \mathrm{O}_{2}$ accounts for $90 \%$ of ROS production under hypoxic conditions and superoxide accounts for $10 \%$ (26). DCF oxidation appears to be a reliable method for the detection of cellular ROS production $(25,27)$. Potential sources of ROS include XO (6), NADPH oxidase (1), the mitochondrial respiratory chain (21), and the metabolic cascade of arachidonic acid (28). XO is the major source of ROS in the rat jugular venous (29) and rat pulmonary circulation (27) following H/R. The mitochondrial respiratory chain is the major source of ROS in embryonic chick cardiomyocytes (21) and human umbilical vein ECs (HUVECs) (30). Thus, we hypothesized that the major source of ROS following $\mathrm{H} / \mathrm{R}$ is both species-specific and organ-specific. When XOR expression was knocked down by XDH siRNA, H/R-induced ROS production in CMECs was also attenuated. $\mathrm{XO}$ accounts for, at least part of, the ROS production induced by $H / R$ in CMECs. 
A

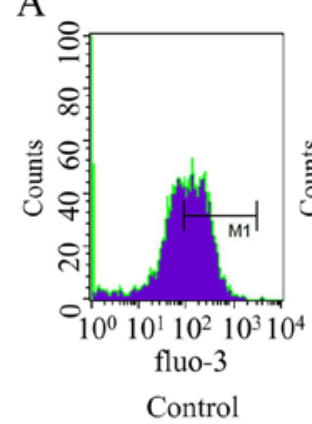

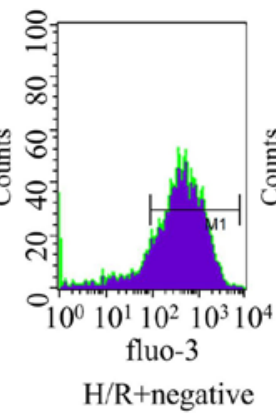

control siRNA

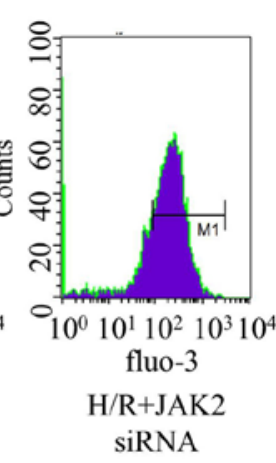

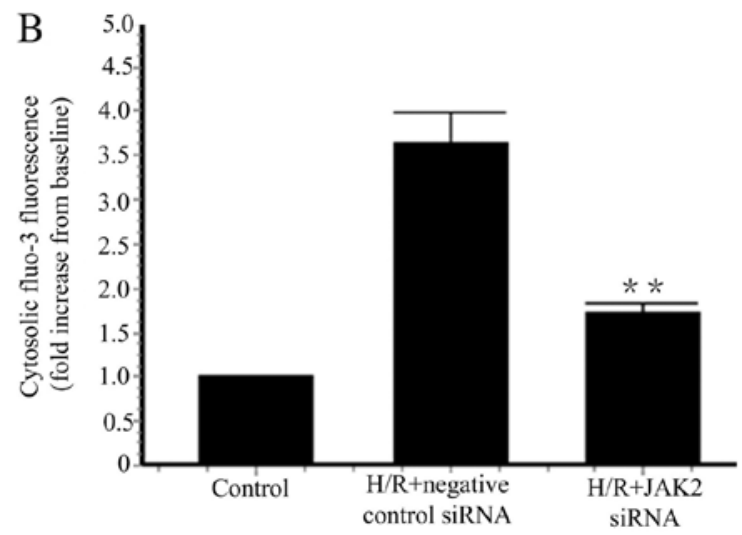

Figure 5. JAK2 siRNA downregulates cytosolic calcium concentrations. (A) Representative data for flow cytometric analysis of fluo-3-stained cardiac microvascular endothelial cells (CMECs) following hypoxia/reoxygenation (H/R). JAK2 siRNA- or negative control siRNA-transfected groups compared with H/R group. (B) Summary data ( $\mathrm{n}=3$ biological replicates) for flow cytometric analysis of fluo-3-stained CMECs after H/R. JAK2 siRNA- or negative control siRNA-transfected group compared with $\mathrm{H} / \mathrm{R}$ group. ${ }^{* *} \mathrm{p}<0.01 \mathrm{vs.} \mathrm{H} / \mathrm{R}$ group.

The signaling pathways involved in the production and activation of XO are controversial under different circumstances in different cell types. p38 MAPK and CK2 have been found to be involved in the activation of $\mathrm{XO}$ following hypoxia in rat pulmonary microvascular ECs (RPMECs) (12). JAKs and STATs are involved in the hypoxia-mediated activation of XO in lung microvascular ECs (LMVECs) (13). The phosphorylation of PI3K increases ROS production during hypoxia in endothelial progenitor cells (31) and in mouse pulmonary microvascular ECs (PMVECs) (25). In the present study, AG490 and LY294002 partially blocked the increase in ROS production following $\mathrm{H} / \mathrm{R}$. The PI3K and JAK2 signaling pathway is significantly activated after $H / R$ whereas the p38 MAPK signaling pathway is unaffected. The pre-treatment of CMECs with AG490 and LY294002 markedly attenuated XO protein levels. The pre-treatment of CMECs with SB203580 did not have the above-mentioned effect. Furthermore, when the knockdown of JAK2 or PI3K was achieved by siRNA, increases in the XO protein levels and ROS production were greatly attenuated. These data show that the PI3K and JAK2 signaling pathways are involved in the upregulation and activation of $\mathrm{XO}$ following H/R. However, HGF inhibits the activation of the JAK2 signaling pathway but not the PI3K signaling pathway. In our previous study, we reported that HGF inhibits the activation and production of $\mathrm{XO}$ by reducing cytosolic $\mathrm{Ca}^{2+}$ concentrations in CMECs after H/R (16). Thus, HGF inhibits XO activation by inhibiting JAK2 signal pathway. In the present study, JAK2 knockdown by JAK2 siRNA significantly reduced cytosolic $\mathrm{Ca}^{2+}$ concentrations. This finding is in agreement with the results of a previous study which demonstrated that AG490, the JAK2 signal inhibitor, blocked an $\mathrm{H}_{2} \mathrm{O}_{2}$-induced increase in intracellular $\mathrm{Ca}^{2+}$ in U937 cells (32). It has been recognised that XO activation is regulated by cytosolic $\mathrm{Ca}^{2+}$, and an unidentified $\mathrm{Ca}^{2+}$-dependent protease is involved in the cleavage of $\mathrm{XDH}$ to $\mathrm{XO}$ (11). A heat-liable protease that cleaves $\mathrm{XDH}$ to $\mathrm{XO}$ has also been found in the mitochondrial intermembrane space (33). In our previous study, when CMECs were pretreated with BAPTA-AM, a cell permeable calcium chelator, the H/R-induced activation of XO was blocked. HGF prevents
JAK2 activation, reduces cytosolic $\mathrm{Ca}^{2+}$ concentrations and in turn, inhibits XO activation in CMECs following H/R.

In conclusion, these findings suggest a novel mechanism whereby HGF regulates $\mathrm{H} / \mathrm{R}$-induced $\mathrm{XO}$ activation in CMECs. The upregulation and activation of $\mathrm{XO}$ as well as increased ROS production following $\mathrm{H} / \mathrm{R}$ primarily involve the PI3K and JAK2 signaling pathways but not the p38 MAPK signaling pathway. HGF inhibits the activation of JAK2. The knockdown of JAK2 attenuated cytosolic $\mathrm{Ca}^{2+}$ concentrations in CMECs following $\mathrm{H} / \mathrm{R}$. Thus, HGF inhibits $\mathrm{XO}$ activation by inhibiting JAK2 activation and reducing cytosolic $\mathrm{Ca}^{2+}$ concentrations in CMECs following H/R. HGF may exhibit protective and therapeutic effects against H/R injury in H/R-related diseases.

\section{Acknowledgements}

The present study was supported by grants (no. 81070185 and 81102079) from the National Natural Science Foundation of China.

\section{References}

1. Yu G, Peng T, Feng Q and Tyml K: Abrupt reoxygenation of microvascular endothelial cells after hypoxia activates ERK1/2 and JNK1, leading to NADPH oxidase-dependent oxidant production. Microcirculation 14: 125-136, 2007.

2. Zhang T, Yang D, Fan Y, Xie P and Li H: Epigallocatechin-3gallate enhances ischemia/reperfusion-induced apoptosis in human umbilical vein endothelial cells via AKT and MAPK pathways. Apoptosis 14: 1245-1254, 2009.

3. Dhar-Mascareño M, Cárcamo JM and Golde DW: Hypoxia-reoxygenation-induced mitochondrial damage and apoptosis in human endothelial cells are inhibited by vitamin $\mathrm{C}$. Free Radic Biol Med 38: 1311-1322, 2005.

4. Pearlstein DP, Ali MH, Mungai PT, Hynes KL, Gewertz BL and Schumacker PT: Role of mitochondrial oxidant generation in endothelial cell responses to hypoxia. Arterioscler Thromb Vasc Biol 22: 566-573, 2002.

5. Jang H-J, Koo BK, Lee HS, Park JB, Kim JH, Seo MK, Yang HM, Park KW, Nam CW, Doh JH and Kim HS: Safety and efficacy of a novel hyperaemic agent, intracoronary nicorandil, for invasive physiological assessments in the cardiac catheterization laboratory. Eur Heart J 34: 2055-2062, 2013.

6. Meneshian A and Bulkley GB: The physiology of endothelial xanthine oxidase: from urate catabolism to reperfusion injury to inflammatory signal transduction. Microcirculation 9: 161-175, 2002. 
7. Landmesser U, Spiekermann S, Preuss C, Sorrentino S, Fischer D, Manes C, Mueller M and Drexler H: Angiotensin II induces endothelial xanthine oxidase activation: role for endothelial dysfunction in patients with coronary disease. Arterioscler Thromb Vasc Biol 27: 943-948, 2007.

8. Spiekermann S, Landmesser U, Dikalov S, Bredt M, Gamez G, Tatge H, Reepschläger N, Hornig B, Drexler H and Harrison DG: Electron spin resonance characterization of vascular xanthine and $\mathrm{NAD}(\mathrm{P}) \mathrm{H}$ oxidase activity in patients with coronary artery disease: Relation to endothelium-dependent vasodilation. Circulation 107: 1383-1389, 2003.

9. Berry CE and Hare JM: Xanthine oxidoreductase and cardiovascular disease: molecular mechanisms and pathophysiological implications. J Physiol 555: 589-606, 2004.

10. Sulikowski T, Domanski L, Ciechanowski K, Adler G, Pawlik A, Safranow K, Dziedziejko V, Chlubek D and Ciechanowicz A: Effect of trimetazidine on xanthine oxidoreductase expression in rat kidney with ischemia-reperfusion injury. Arch Med Res 39: 459-462, 2008

11. McNally JS, Saxena A, Cai H, Dikalov S and Harrison DG: Regulation of xanthine oxidoreductase protein expression by hydrogen peroxide and calcium. Arterioscler Thromb Vasc Biol 25: 1623-1628, 2005.

12. Kayyali US, Donaldson C, Huang H, Abdelnour R and Hassoun PM: Phosphorylation of xanthine dehydrogenase/oxidase in hypoxia. J Biol Chem 276: 14359-14365, 2001.

13. Wang G, Qian P, Jackson FR, Qian G and Wu G: Sequential activation of JAKs, STATs and xanthine dehydrogenase/oxidase by hypoxia in lung microvascular endothelial cells. Int J Biochem Cell Biol 40: 461-470, 2008.

14. Reviriego-Mendoza MM and Santy LC: The cytohesin guanosine exchange factors (GEFs) are required to promote HGF-mediated renal recovery after acute kidney injury (AKI) in mice. Physiol Rep 3: e12442, 2015.

15. White HM, Acton AJ, Kamocka MM and Considine RV: Hepatocyte growth factor regulates neovascularization in developing fat pads. Am J Physiol Endocrinol Metab 306 E189-E196, 2014.

16. Zhang Y, Hu S and Chen Y: Hepatocyte growth factor suppresses hypoxia/reoxygenationinduced XO activation in cardiac microvascular endothelial cells. Heart Vessels 30: 534-544, 2015

17. Nishida M, Carley WW, Gerritsen ME, Ellingsen O, Kelly RA and Smith TW: Isolation and characterization of human and rat cardiac microvascular endothelial cells. Am J Physiol 264: H639-H652, 1993

18. Zhang Z, Li W, Sun D, Zhao L, Zhang R, Wang Y, Zhou X, Wang $\mathrm{H}$ and Cao F: Toll-like receptor 4 signaling in dysfunction of cardiac microvascular endothelial cells under hypoxia/reoxygenation. Inflamm Res 60: 37-45, 2011.

19. Ladilov Y, Schäfer C, Held A, Schäfer M, Noll T and Piper HM: Mechanism of $\mathrm{Ca}(2+)$ overload in endothelial cells exposed to simulated ischemia. Cardiovasc Res 47: 394-403, 2000.
20. Yu G, Bolon M, Laird DW and Tyml K: Hypoxia and reoxygenation-induced oxidant production increase in microvascular endothelial cells depends on connexin40. Free Radic Biol Med 49: 1008-1013, 2010.

21. Loor G, Kondapalli J, Iwase H, Chandel NS, Waypa GB, Guzy RD, Vanden Hoek TL and Schumacker PT: Mitochondrial oxidant stress triggers cell death in simulated ischemia-reperfusion. Biochim Biophys Acta 1813: 1382-1394, 2011.

22. Kong R, Jia G, Cheng ZX, Wang YW, Mu M, Wang SJ, Pan SH, Gao Y, Jiang HC, Dong DL and Sun B: Dihydroartemisinin enhances Apo2L/TRAIL-mediated apoptosis in pancreatic cancer cells via ROS-mediated up-regulation of death receptor 5. PLoS One 7: e37222, 2012.

23. Przygodzki T, Sokal A and Bryszewska M: Calcium ionophore A23187 action on cardiac myocytes is accompanied by enhanced production of reactive oxygen species. Biochim Biophys Acta 1740: 481-488, 2005.

24. Rasband WS (ed): ImageJ. U.S. National Institutes of Health, Bethesda, MD, 1997-2012.

25. Chatterjee S, Browning EA, Hong N, DeBolt K, Sorokina EM, Liu W, Birnbaum MJ and Fisher AB: Membrane depolarization is the trigger for PI3K/Akt activation and leads to the generation of ROS. Am J Physiol Heart Circ Physiol 302: H105-H114, 2012.

26. Kelley EE, Khoo NK, Hundley NJ, Malik UZ, Freeman BA and Tarpey MM: Hydrogen peroxide is the major oxidant product of xanthine oxidase. Free Radic Biol Med 48: 493-498, 2010.

27. Saito S, Ogawa J and Minamiya Y: Pulmonary reexpansion causes xanthine oxidase-induced apoptosis in rat lung. Am J Physiol Lung Cell Mol Physiol 289: L400-L406, 2005.

28. Krause GS, White BC, Aust SD, Nayini NR and Kumar K: Brain cell death following ischemia and reperfusion: A proposed biochemical sequence. Crit Care Med 16: 714-726, 1988.

29. Ono T, Tsuruta R, Fujita M, Aki HS, Kutsuna S, Kawamura Y, Wakatsuki J, Aoki T, Kobayashi C, Kasaoka S, et al: Xanthine oxidase is one of the major sources of superoxide anion radicals in blood after reperfusion in rats with forebrain ischemia/reperfusion. Brain Res 1305: 158-167, 2009.

30. Therade-Matharan S, Laemmel E, Duranteau J and Vicaut E Reoxygenation after hypoxia and glucose depletion causes reactive oxygen species production by mitochondria in HUVEC. Am J Physiol Regul Integr Comp Physiol 287: R1037-R1043, 2004.

31. Dai T, Zheng H and Fu GS: Hypoxia confers protection against apoptosis via the PI3K/Akt pathway in endothelial progenitor cells. Acta Pharmacol Sin 29: 1425-1431, 2008

32. Shimizu S, Yonezawa R, Hagiwara T, Yoshida T, Takahashi N, Hamano S, Negoro T, Toda T, Wakamori M, Mori Y and Ishii M: Inhibitory effects of AG490 on $\mathrm{H}_{2} \mathrm{O}_{2}$-induced TRPM2-mediated $\mathrm{Ca}(2+)$ entry. Eur J Pharmacol 742: 22-30, 2014.

33. Saksela M, Lapatto R and Raivio KO: Irreversible conversion of xanthine dehydrogenase into xanthine oxidase by a mitochondrial protease. FEBS Lett 443: 117-120, 1999. 